\title{
Cerebral blood flow velocities modulate during cognitive tasks using vision and vision-like requirements: A TCD study
}

\author{
APRIL HORTON-LAMBIRTH and ALBERT E. ROBERTS \\ Catawba College, Salisbury, North Carolina \\ and \\ WILLJAM M. MCKINNEY \\ Bowman-Gray School of Medicine, Winston-Salem, North Carolina
}

\begin{abstract}
Blood flow velocities in the anterior, middle, and posterior cerebral arteries (VACAs, VMCAs, VPCAs) of three separate groups of healthy volunteers $(n=22-24)$ were obtained noninvasively with a transcranial Doppler procedure during rest and during sets of 24-sec cognitive tasks that had varied visual requirements: remembering seen and heard items; generating autobiographical images; and visualizing and mentally rotating stimuli. Both global and time-course velocity values (averaged and for each of eight measures, respectively, from a thinking period) were analyzed. Global VMCA increased bilaterally over baseline during recalling; global VACA was not affected; and global VPCA increased after the subjects saw but not after they heard the items. Global VMCA and VACA elevated bilaterally during imaging, but VPCA did not. Visualizing, but not mentally rotating, stimuli increased all three global velocities bilaterally. Task-dependent time-course modulations in velocities appeared during the thinking periods. Velocities hyperelevated temporarily at the outset of all periods, except for VPCA during tasks not requiring direct vision. VACA slowed to baseline during recollection of the heard items, and all velocities slowed during the visualizing tasks but remained above baseline.
\end{abstract}

The noninvasive transcranial Doppler (TCD) neuroimaging technique uses ultrasound to measure blood flow velocity $(\mathrm{V})$ in the cerebral arteries. TCD measures make a recognized contribution in the assessment of intracranial cerebrovascular dysfunctions (see Reutern \& Büdingen, 1993), and a new use is emerging for TCD neuroimaging: documenting changes in velocity measures while healthy persons perform cognitive activities (Droste, Harders, \& Rastogi, 1989a, 1989b; Harders, Laborde, Droste, \& Rastogi, 1989; O'Dell, Roberts, \& McKinney, 1992a, 1992b). In this line of research, a cerebral artery (usually the middle cerebral artery, MCA) is sampled. TCD measures taken while persons are at rest (baseline, BL) then are compared to measures obtained while they comply with the requirements of the cognitive probe. Compared with VBL, VMCA typically increases bilaterally during thinking, generally by $3 \%-6 \%$ (see Droste et al., 1989a; O'Dell et al., 1992b), although hemisphere differences sometimes occur (Droste et al., 1989b, Experiment 1; Hartje, Ringelstein, Kistinger, Fabianek, \& Willmes, 1994).

The velocity data provided by TCD neuroimaging can be examined as global values or as time-course patterns. The former is an average of the sets of measures obtained

This research was supported by Grant NS31774 from the National Institute of Neurological Disorders and Stroke (to A.E.R.). Reprint requests and correspondence should be addressed to A. E. Roberts, Department of Psychology, Catawba College, Salisbury, NC 28144 (e-mail: aroberts@catawba.edu). during the thinking period and shows the aggregate effect of a cognitive task on velocity. The latter maintains the relative positions of the separate measures within each recording interval of the period so as to reveal modulations in velocity that occur as persons comply with the cognitive requirement. A marked advantage of the TCD procedure is the ability to provide these latter measures in real time, because dynamic changes in velocity often occur while persons think. For example, the Droste and Harders's studies demonstrated that VMCA may hyperelevate temporarily over VBL early in the thinking period. Moreover, if the task requirement is completed before the prescribed thinking period expires, it is likely that VMCA will slow and match VBL (O'Dell et al., 1992a; Schmelzeis, Roberts, \& McKinney, 1994).

The TCD research protocol now includes other cerebral arteries (e.g., the anterior and posterior cerebral arteries; ACAs and PCAs, respectively) from the same (Kelley et al., 1992) or different (Schmelzeis et al., 1994; Varnadore, Roberts, \& McKinney, 1997a, 1997b) persons while they engage in identical tasks. This research shows that changes in velocity often are task dependent. For example, a visual-motor task increases VMCA more than it does both VACA and, in the right hemisphere, VPCA (Kelley et al., 1992). Short-term remembering, decisionmaking, and problem-solving tasks elevate both VMCA and VACA but not VPCA (Schmelzeis et al., 1994).

The cognitive tasks incorporated into TCD research protocols include passive receptions of inputs (Harders et al., 
1989; Kelley, Chang, Suzuki, Levin, \& Reyes-Iglesias, 1993; Njemanze, 1991; Njemanze, Gomez, \& Horenstein, 1992), interactions between sensory-cognitive-motor requirements (Droste et al., 1989a, 1989b; Kelley et al., 1992; Silvestrini, Cupini, Matteis, Troisi, \& Caltagirone, 1994), and exclusively generative mentations (Markus \& Boland, 1992; O'Dell et al., 1992a, 1992b; Schmelzeis et al., 1994; Silvestrini et al., 1994). Inasmuch as a cognitive task involves a greater number of interactive processing requirements, cortical involvements become more complex. For example, positron emission tomography (PET) shows that, when subjects imagine their intended hand movements, the pattern of both cortical and subcortical brain activations broaden, as compared with when they are passively watching those hand movements (Decety et al., 1994). Even the mental processing of a single word engages multiple and widely separated regions of the cerebral cortex, depending on the processing requirement (Miller, 1995, Figure 4; Petersen, Fox, Posner, Mintun, \& Raichle, 1988). As persons comply with interactive cognitive requirements, separate sites irrigated by a common arterial source (e.g., the MCA) are activated and constrain the spatial resolution capability of TCD neuroimaging. However, the duration of specific activations probably are temporally finite, and activation effects should covary with the time-course measures provided by TCD neuroimaging.

Our study was guided by two purposes: (1) to compare changes in the velocities of three cerebral arteries during sets of cognitive tasks and (2) to examine the influence of the different task requirements on both global and timecourse velocity measures. To accomplish these purposes, we measured VMCA, VACA, and VPCA from different groups of healthy persons during cognitive activities for which the visual requirements varied: remembering previously seen items, generating and maintaining an image of a personal experience (autobiographical imaging), reading and studying (visualizing) visual items, and creating and rotating images of previously seen items (e.g., envisaging). Our cognitive probes were drawn from a visual modality because (1) velocity in these cerebral arteries is responsive to vision-based tasks, (2) single task probes can be arranged to engage mentation throughout the recording period, and (3) variations in task requirements can be accomplished while accompanying expressive and motor activities are minimized.

\section{METHOD}

\section{Subjects and Equipment}

After giving informed consent, 68 college students (mean age = 20 years; range $=18-24.5$ years) served in an MCA $(n=22)$, ACA $(n=22)$, or PCA $(n=24)$ group. Each group had 8 men, and all were experimentally naive about the TCD procedure, reported no medically diagnosed problems, and were medication free. All smokers were abstinent for at least $1 \mathrm{~h}$ prior to the experiment.

A TC2-64B TCD (Nicolet/EME, Madison, WI) measured blood flow velocity. Each cerebral artery was accessed through the temporal window (Aaslid, 1986), with the MCA insonated at a depth of $50 \mathrm{~mm}$, the ACA at $60-65 \mathrm{~mm}$, and the $\mathrm{P}_{2}-$ segment of the PCA at $65 \mathrm{~mm}$, each with an ultrasound intensity of $100 \mathrm{~mW} / \mathrm{cm}^{2}$. The 2-MHz transducer (Nicolet/EME, IMP2 monitoring probe) was secured in a fixation plate strapped to the head in order to permit continuous measurement, even in the presence of any head movements. Time-averaged mean velocity values were obtained from the TCD unit every $3 \mathrm{sec}$.

\section{Procedure}

Each person was tested while in a reclining chair located in a dimly lit, sound-attenuated room and, unless instructed differently, performed with his or her eyes closed. After insonating an artery, the audio signal from the TCD unit was turned off, and a 5-min rest period began, the final $60 \mathrm{sec}$ of which provided the BL measure for the first experimental phase. Three consecutive phases (with one to three cognitive tasks, each given once) were presented, and each phase was preceded by a 60 -sec $\mathrm{BL}$ period. These phases incorporated tasks, between which the visual requirements were varied as described below. A final 60 -sec BL ensued after the third phase. The mentation period for all tasks was $24 \mathrm{sec}$, in order to minimize performance artifacts-for example, mental fatigue, attention drifts.

The sequence of three phases was given twice, once under leftside and once under right-side insonation. The side first insonated was counterbalanced between persons. The task probes in the second insonation were similar to those in the first, and the probes were counterbalanced between sides. Velocity measures were not recorded when the subjects received instructions or when they gave answers to the probes, and the subjects were not permitted to ask questions during the thinking periods.

The recalling phase consisted of two tasks that required the person to remember two different sets of four consonants and a numberfor example, D K $5 \mathrm{G} \mathrm{J}$. One set was shown on a $3 \times 5 \mathrm{in}$. card for $3 \mathrm{sec}$, and the other set was read by the experimenter. This arrangement permitted the effects of visual and verbal presentations of the task on velocity measures to be compared. The order of presentation was counterbalanced within a group.

In the imaging phase, each person generated and maintained an autobiographical image (e.g., of their high school graduation) in order to study the effect of an envisioning activity on velocities. Recording began when the person raised the little finger on his or her right hand to signal that the target image had been generated.

The visualizing/envisaging phase consisted of three separate tasks. The person first silently reviewed a card having five items (e.g., R F 2 B K) in order to be familiar with the items. Next, the person silently studied a card (active viewing) with a new set of five items so that they could recognize the characters if later presented backward and/or rotated. In the first task, the effect of looking at stimuli (passive viewing) on velocities was assessed; in the second task, it was determined whether active viewing had effects different from passive viewing; and both tasks served to uncover possible effects that were due to attention drifts within the thinking period. The envisaging task was adapted from the mental rotation procedure of Cooper and Shepard (1973). The person viewed a card consisting of five items and was told that two of the items would be changed on a subsequent card-that is, rotated, printed backward, or both. The person then envisaged and mentally varied the items during the thinking period, with eyes closed, so that changes to items on a card (shown at the end of the period) could be identified quickly. Identifications were recorded as correct or incorrect in order to encourage compliance with the envisaging requirement.

\section{Statistical Analyses}

The effects of the cognitive tasks on velocities were analyzed in terms of singular changes (separate analyses of the velocities in each artery) and comparative changes, which directly compared velocity changes between the three arteries.

Singular changes. Global velocity changes induced by the tasks were assessed by calculating a mean velocity for each of the tasks 
Table 1

Bilaterally Averaged (Global) Blood Flow Velocities (in Centimeters/Second) in the MCA, ACA and PCA Obtained During the Three Phases of the Experiment

\begin{tabular}{|c|c|c|c|c|c|c|c|c|c|c|}
\hline & \multicolumn{10}{|c|}{ Experimental Phases } \\
\hline & \multicolumn{3}{|c|}{ Recalling } & \multirow[b]{2}{*}{$\mathrm{BL}$} & \multirow[b]{2}{*}{ Imaging } & \multicolumn{4}{|c|}{ Visualizing/Envisaging } & \multirow[b]{2}{*}{ BL-T } \\
\hline & $\mathrm{BL}$ & Heard & Seen & & & BL & Review & Study & Envisaging & \\
\hline \multicolumn{11}{|c|}{ Bilateral VMCA $(n=22)$} \\
\hline$M$ & 54.9 & 56.7 & 57.7 & 54.0 & 56.2 & 55.4 & 57.1 & 57.4 & 56.1 & 55.9 \\
\hline$S D$ & 9.9 & 10.6 & 10.2 & 10.1 & 10.6 & 10.6 & 10.9 & 10.9 & 10.6 & 11.8 \\
\hline$\%$ & & $3.3 \%$ & $5.1 \%$ & & $4.1 \%$ & & $3.1 \%$ & $3.6 \%$ & n.s. & \\
\hline \multicolumn{11}{|c|}{ Bilateral VACA $(n=22)$} \\
\hline$M$ & 49.2 & 49.9 & 49.5 & 48.0 & 49.5 & 48.0 & 49.7 & 49.4 & 48.0 & 47.6 \\
\hline$S D$ & 8.0 & 8.2 & 8.5 & 8.4 & 8.8 & 8.5 & 9.2 & 9.3 & 8.9 & 9.3 \\
\hline$\%$ & & n.s. & n.s. & & $3.1 \%$ & & $3.5 \%$ & $2.9 \%$ & n.s. & \\
\hline \multicolumn{11}{|c|}{ Bilateral VPCA $(n=24)$} \\
\hline$M$ & 35.2 & 34.4 & 36.0 & 34.9 & 34.4 & 34.9 & 37.4 & 37.6 & 34.5 & 35.3 \\
\hline$S D$ & 8.8 & 8.7 & 9.7 & 9.5 & 9.2 & 9.1 & 10.1 & 9.8 & 9.8 & 9.2 \\
\hline$\%$ & & n.s. & $2.3 \%$ & & n.s. & & $7.2 \%$ & $7.7 \%$ & n.s. & \\
\hline
\end{tabular}

Note-BL-T, final baseline period given at completion of the tasks.

(on the basis of the eight velocity measures obtained during a mentation period) for each person. The final $30 \mathrm{sec}$ of a BL provided a single mean value for that $B L$. These mean data were analyzed by separate (for MCA, ACA, and PCA) two-way analyses of variance (ANOVAs), with repeated measures on insonation side (left and right) and the tasks within a phase (including the BL).

Time-course velocity changes in each artery during mentating also were examined by comparing the velocities of each person in each of the eight mentation intervals with the VBL by separate (for MCA, ACA, and PCA) repeated measures ANOVAs.

Comparative changes. Relative changes in velocities between the three arteries during tasks were compared directly by calculating difference scores (Wainer, 1991) for each person (e.g., $V_{\text {interval }}-$ $V_{\mathrm{BL}}$ ) for each of the eight mentation intervals of a task. These scores were analyzed by separate 3 (MCA, ACA, PCA) $\times 8$ (repeated measures on the mentation intervals) mixed ANOVAs. This analysis compared both the relative global changes in arterial velocities and relative time-course changes in velocities during a $24-\mathrm{sec}$ mentating period.

Newman-Keuls tests were used for all post hoc multiple comparisons. Alpha levels were set at .05 for all statistical analyses.

\section{RESULTS}

With the ANOVAs on the global velocities it was found that differences attributable to either the side insonated or a side $\times$ task interaction were not significant for any velocity measure (all $F \mathbf{s}<2$ ). Separate ANOVAs performed on VBL values of the three groups confirmed that VBL did not change significantly between experimental phases (all $F$ s $<2$ ). Thus, the results provided below are given in terms of bilaterally averaged data. The effects of tasks on the global velocities of each artery are shown in Table 1, and degrees of change from BL during the 24-sec mentation periods (time-course changes) appear in Figures 1-3, with artery comparisons summarized in Table 2.

\section{Velocity Changes During Recalling}

Specific changes. The $2 \times 3$ ANOVAs on the global mean velocities summarized in Table 1 showed that VMCA was faster in the recall period after the items were seen than it was after the items were heard, and that both were elevated over BL $[F(2,42)=26.24, p<.001]$, and that no significant differences were obtained for VACA (all $F$ s $<1.5$ ). VPCA was faster after the items were seen than it was either after they were heard or at BL $[F(2,46)=$ $3.69, p<.05$ ], and differences between the latter two were not significant.

Velocity measures from all three arteries modulated across the recalling period after the items were seen. VMCA increased over BL in the first $9 \mathrm{sec}$ and in the final $9 \mathrm{sec}$ of this period, whereas both VACA and VPCA were faster than their BLs only in the first 6 sec. After the items were heard, both VMCA and VACA increased significantly over their BLs in the first $6 \mathrm{sec}$, but both then slowed to match their BL; VMCA again was faster than $\mathrm{BL}$ in the final $6 \mathrm{sec}$. VPCA did not differ significantly from its $\mathrm{BL}$ during any interval.

Comparative changes. After the items were seen, global VMCA was more elevated than were global VACA and VPCA $[F(2,65)=7.64, p<.025]$. However, VMCA and VACA increased comparably after the items were heard, and both were faster than VPCA $[F(2,65)=10.08$, $p<.001]$.

Arterial differences in velocity modulations (Figure 1) were confirmed statistically $[F(7,455)=50.14, p<.001]$. After the items were seen, VMCA was faster in the first $6 \mathrm{sec}$ (Intervals 1 and 2) than were VACA and VPCA. These differences disappeared during the middle of the recalling period but reemerged in the final $9 \mathrm{sec}$ (Intervals $6-8$ ). But, after the items were heard, VMCA and VACA were elevated over BLs comparably in the first $12 \mathrm{sec}$ (Intervals 1-4), and each more so than VPCA. The degree of elevation in VACA lessened and matched VPCA in the final $12 \mathrm{sec}$, and both were less effected than was VMCA in these latter intervals.

\section{Velocity Changes During Imaging}

Singular changes. All subjects reported success in generating the required images. The $2 \times 2$ ANOVAs on 


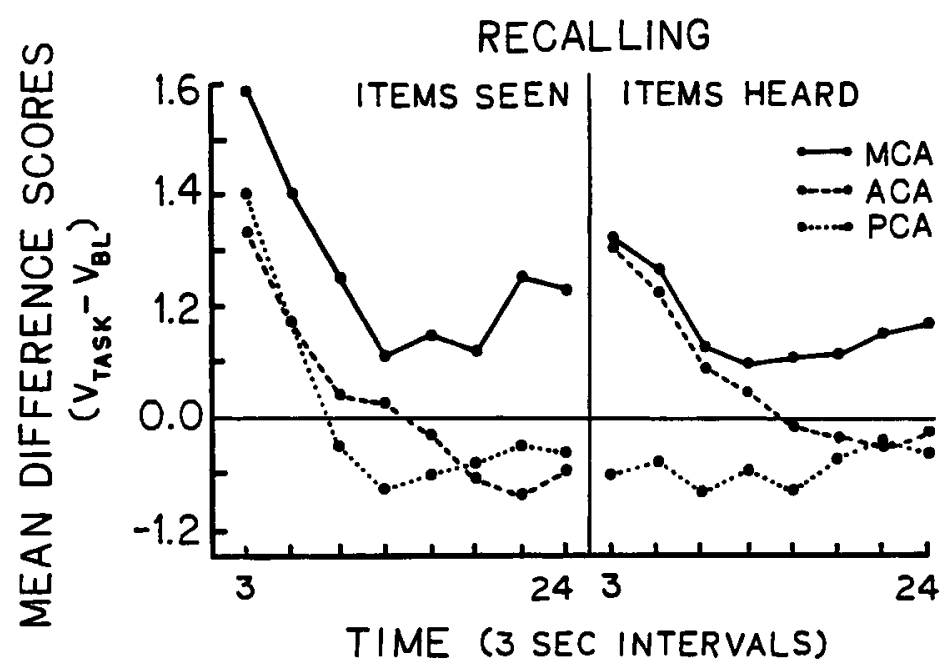

Figure 1. Time course changes in bilateral blood flow velocity $\left(V_{\text {task }}-V_{B L}\right)$ while recalling stimuli presented on a card (items seen) and stimuli read by the experimenter (items heard). Velocity data are for the three MCA, ACA, and PCA groups.

the global mean velocities showed that VMCA and VACA increased significantly over their BLs $[F \mathrm{~s}(1,21)=21.94$ and 16.59 , respectively, $p s<.001]$, but that VPCA did not $[F(1,23)=1.49$, n.s.]. Both VMCA and VACA were significantly faster than their BLs in the first $9 \mathrm{sec}$ of the imaging period, but VMCA also was significantly faster in the final $9 \mathrm{sec}$. As Figure 2 suggests, VPCA did not differ significantly from $B L$ in any interval.

Comparative changes. The degrees of change from BL for global VMCA and VACA were not statistically different, and both were enhancive, compared with that for VPCA $[F(2,65)=10.86, p<.001]$. The degrees of en-

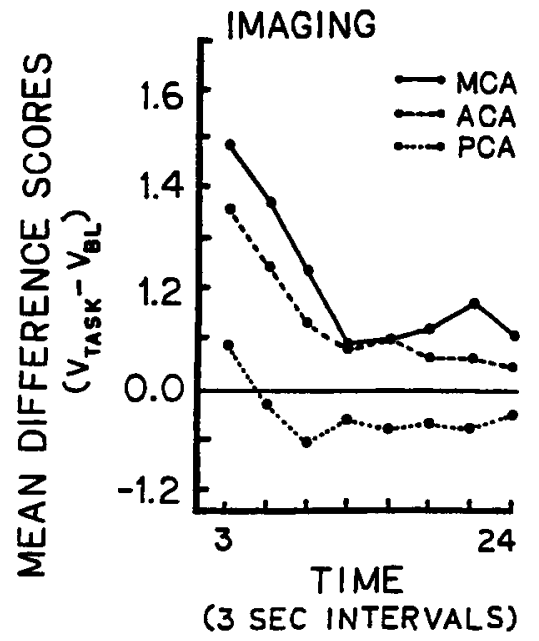

Figure 2. Time course changes in bilateral blood flow velocity $\left(V_{\text {task }}-V_{B L}\right)$ while generating and maintaining an image of a personal experience. Velocity data are for the three MCA, ACA, and PCA groups. hancive change for VMCA and VACA in the first $9 \mathrm{sec}$ were not significantly different, and both slowed and matched VPCA. In the final $6 \mathrm{sec}$, however, VMCA was more elevated over its BL than was either VACA or VPCA.

\section{Velocity Changes During \\ Visualizing and Envisaging}

Singular changes. The global mean velocities for all three arteries increased significantly over BL during the review and study tasks (all $F \mathrm{~s}>8.50$, all $p \mathrm{~s}<.001$ ). The mental rotation task did not produce significant differences in the global mean velocities in any of the three arteries (all $F \mathrm{~s}<1$ ).

During the review of target items, VMCA and VACA each was elevated over its BL in all but the last $3 \mathrm{sec}$, whereas VPCA was elevated over its BL throughout the period. Both VMCA and VPCA were faster than their BLs throughout the study period, but VACA was faster than its BL only during the first $12 \mathrm{sec}$. Finally, VMCA increased over BL during the first $9 \mathrm{sec}$ of the envisaging period, whereas VACA and VPCA were faster only during the first $3 \mathrm{sec}$ and then slowed significantly below their BLs during the final $9 \mathrm{sec}$.

Comparative changes. Arterial differences in the global degrees of change from BL during the three tasks (Figure 3 ) were statistically nonsignificant $\left(F_{\mathrm{S}}=1.18\right.$, 1.80 , and 2.13, respectively; all n.s.). Moreover, the degrees of change in the three velocities from their BLs in each interval of each of these three tasks were statistically comparable (all $F \mathrm{~s}<1$ ).

\section{DISCUSSION}

Our study underscores the merit of following a research protocol that (1) samples more than one cerebral 


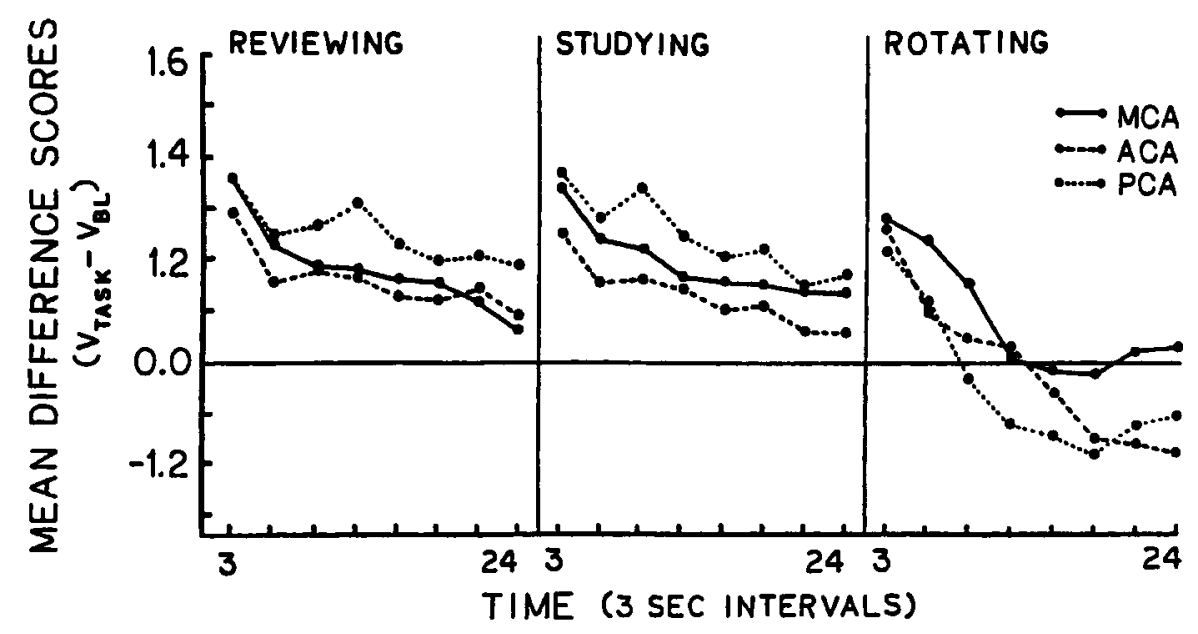

Figure 3. Time course changes in bilateral blood flow velocity $\left(V_{\text {task }}-V_{B L}\right)$ during the two direct visualizing activities (reviewing and studying stimuli) and the envisaging activity (mental rotation). Velocity data are for the three MCA, ACA, and PCA groups.

artery and (2) obtains time-course velocity patterns while persons mentate. With respect to the former, changes in the three velocities were task dependent. The recall tasks had the strongest effect on global VMCA and only nominally affected VACA. Changes in global VPCA depended on how the items-to-be-remembered were given: global VPCA was elevated during recollection of items given visually but not during recollection of listened-to items. This relative lack of responsiveness in VPCA after items were heard is consistent with reports examining rCBF (Posner, Petersen, Fox, \& Raichle, 1988). The imaging task increased both global VMCA and VACA, but global VPCA was relatively unaffected. Finally, the two silent visualizing tasks (reviewing and studying stimuli) elevated all three global velocities comparably, but the envisaging task exerted no significant effect on the global velocity measures.

With respect to the time-course velocity patterns, velocities modulated within the 24-sec mentation periods, so the global values were not always based on steady-state indices. For example, although global VACA and VBL were not significantly different, VACA actually accelerated longer into the recalling period after the items were heard than it was after the items were seen, before slowing to match VBL. In addition, VMCA showed accelerative rebound in the later portions of the two recalling periods as well as during the imaging period, and the global values, although enhancive, masked these dynamics. Accelerative rebounds also are present in the early portions of longer mentation periods (see Droste et al., 1989a, 1989b; Harders et al., 1989); the factors controlling such rebounds have yet to be identified.

The time-course data for the envisaging task, given in Figure 3, suggest that this task may not have sustained mentation. We expanded the mental rotation requirement in order to induce extended mentation, but the task was resolved quickly (consistent with the results of Cooper \& Shepard, 1973). With minimal additional mentation being needed, velocities slowed (Schmelzeis et al., 1994). In retrospect, a mental rotation requirement that was less reliant on envisaging may have been more suitable-for example, rotating three-dimensional patterns to match a

Table 2

Pairs Comparisons of the Degrees of

Acceleration in Global Velocities of the Three Cerebral Arteries Within Each Cognitive Task

\begin{tabular}{lccc}
\hline & \multicolumn{3}{c}{ Velocity Comparisons } \\
\cline { 2 - 4 } Cognitive Task & VMCA vs. VACA & VMCA vs. VPCA & VACA vs. VPCA \\
\hline Recalling & $p<.025$ & $p<.025$ & n.s. \\
Items seen & n.s. & $p<.001$ & $p<.001$ \\
Items heard & n.s. & $p<.001$ & $p<.001$ \\
Imaging & & & \\
$\quad$ Visualizing & n.s. & n.s. & n.s. \\
Viewing & n.s. & n.s. & n.s. \\
Studying & n.s. & n.s. & n.s. \\
Envisaging & n.
\end{tabular}

Note-When significant differences are noted, the degree of acceleration from baseline was more for the first member of the pair. 
target (Vandenberg \& Kuse, 1978). The sources governing the slowing in VACA and VPCA below VBL later in the envisaging period are unclear.

Changes in blood flow velocity measures point to correlated changes in cerebral blood flow (Bishop, Powell, Rutt, \& Browse, 1986; Dahl, Russell, Nyberg-Hansen, \& Rootwelt, 1992) and thus reflect changes in brain activations that occurred coincident with cognitive-processing requirements. However, physiologic factors external to cognitive probes potentially can affect cerebral hemodynamics (see Reutern \& Büdingen, 1993)-for example, changes in respiration, heart rate, blood pressure, metabolic byproducts (expiratory pCO2). Evidence suggests that these factors have a benign influence in TCDcognition studies (Bulla-Hellwig, Vollmer, Götzen, Skreczek, \& Hartje, 1996; Cupini et al., 1996; Silvestrini et al., 1994) and so they were not assessed. The gradual lessening in velocity during the passive and active visualizing tasks, although elevated over BLs through most of the 24-sec periods, likely reflects attention drifts stemming from the processing of simple probe elements.

The MCA irrigates temporal lobe areas, and global VMCA was quite responsive to language-based memoryrelated tasks, more so than were the other two velocities. The responsiveness of global VACA to these tasks, though undistinguished, is in line with other studies in this respect (Kelley et al., 1992; Schmelzeis et al., 1994; Varnadore et al., 1997a, 1997b). The ACA serves regions associated with attention mechanisms (Petersen et al., 1988; Posner et al., 1988), and elevation of VACA may coincide with the cognitive engagement of these mechanisms and diminish as information-processing requirements shift - for example, from attending to recalling or comprehending. The gradual slowing of VACA after items-to-be-remembered were heard points to the possibility that attention mechanisms activated for listened-to stimuli differ from those engaged when attending to visual inputs. This possibility gains support from Knecht et al., (1996), who used a language task to isolate an attentiveness component at the beginning of biphasic time-course velocity patterns.

The PCA perfuses primary visual regions of the brain, and VPCA was affected strongly by visualizing activities but not by activities for which stimuli were not concurrently available for viewing - that is, imaging. Visualizing and imaging activities often are viewed as calling upon common mechanisms for their execution (Farah, 1988; Tippett, 1992), but our results suggest that it is likely that the imaging probe we provided (based on an episodic experience) called upon memorial rather than visual mechanisms in the brain (see Tulving, 1989) - that is, the persons generated a vivid memory.

The real-time TCD velocity measures offer an opportunity to monitor the rapid changes in brain dynamics that occur coincident with mentating, as they happen in contrast to postactivation assessment procedures - for example, PET, fMRI. Moreover, the time-course velocity measures provided by TCD neuroimaging also present a picture of modulations in hemovelocity not available from single-value indices. These time-course velocity patterns also show that some cognitive happenings (our mental rotation requirement) may occur more rapidly than can be captured, even by TCD neuroimaging.

Table 1 shows rather large between-subjects dispersions in our VBL measures, but large standard deviations (SDs) typically accompany group means (see Sorteberg, Langmoen, Lindegaard, \& Nornes, 1990). Moreover, the percent increases in velocity shown in Table 1 compare favorably with other reports on the effects of thinking tasks on cerebral blood flow velocity (cf. Droste, Harders, \& Liberti, 1996; Hartje et al., 1994) but may appear relatively small in light of the group $S D$ s. A reason for this paradox is that between-subjects differences typify steadystate velocity measures, but within-subjects variability in measures does not: The $S D$ s for our subjects' VBL values were less than $2 \%$ of their global VMCA and VACA means and $3.5 \%$ of their global VPCA means. Thus, it is likely that task-induced changes in velocity outside of this BL range represent reliable effects. Our interartery comparisons should be viewed with caution until replicated with procedures that measure the three velocities in the arteries of the same person. TCD technology now is available for obtaining simultaneous bilateral velocity measures (Droste et al., 1996; Rihs et al., 1995), thus (1) making successive hemispheric insonations unnecessary, (2) shortening the testing period, and, thereby, (3) increasing the feasibility of using within-subjects designs to insonate more than one cerebral artery from the same subject.

\section{REFERENCES}

AASLID, R. (1986). Transcranial Doppler examination techniques. In R. Aaslid (Ed.), Transcranial Doppler sonography (pp. 39-59). New York: Springer-Verlag.

Bishop, C. C. R., Powele, S., RutT, D., \& Browse, N. L. (1986). Transcranial Doppler measurement of middle cerebral artery blood flow velocity: A validation study. Stroke, 17, 913-915.

Bulla-Hellwig, M., Vollmer, J., Götzen, A., Skreczek, W., \& HARTJE, W. (1996). Hemispheric asymmetry of arterial blood flow velocity changes during verbal and visuospatial tasks. Neuropsychologia, 34, 987-991.

COOPER, L. A., \& ShePard, R. N. (1973). The time required to prepare for a rotated stimulus. Memory \& Cognition, 1, 246-250.

Cupini, L. M., Matteis, M., Triosi, E., Sabbadini, M., Bernardi, G., Caltagirone, C., \& Silvestrini, M. (1996). Bilateral simultaneous transcranial Dopper monitoring of flow velocity changes during visuospatial and verbal working memory tasks. Brain, 119, 1249-1253.

Dahl, A., Russell, D., Nyberg-Hansen, R., \& Rootwelt, K. (1992). A comparison of regional cerebral blood flow and middle cerebral artery blood flow velocities: Simultaneous measurements in healthy subjects. Journal of Cerebral Blood Flow \& Metabolism, 12, 1049 1054.

Decety, J., Perani, D., Jeannerod, M., Bettinardi, V., Tadary, B., Woods, R., MAzzIOTTA, J. C., \& FAZIO, F. (1994). Mapping motor representations with positron emission tomography. Nature, 371, 600-602.

Droste, D. W., Harders, A. G., \& LiberTi. G. (1996). Bilateral simultaneous TCD monitoring during the performance of a verbal fluency task and a face recognition task. Journal of Psychophysiology, 10, 303-309.

Droste, D. W., Harders, A. G., \& Rastogi, E. (1989a). A TCD study of blood flow velocity in the middle cerebral arteries performed at rest and during mental activities. Stroke, 20, 1005-1011. 
Droste, D. W., Harders, A. G., \& Rastogi, E. (1989b). Two transcranial studies on blood flow velocity in both middle cerebral arteries during rest and the performance of cognitive tasks. Neuropsychologia, 27, 1221-1230.

FaraH, M. J. (1988). Is visual imagery really visual? Overlooked evidence from neuropsychology. Psychological Review, 95, 307-317.

harders, A. G., Laborde, G., Droste, D. W., \& Rastogi, E. (1989). Brain activity and blood flow velocity changes: A transcranial Doppler study. International Journal of Neuroscience, 47, 91-102.

Hartje, W., Ringelstein, E. B., Kistinger, B., Fabianek, D., \& WILLMES, K. (1994). Transcranial Doppler ultrasonic assessment of middle cerebral artery blood flow velocity changes during verbal and visuospatial cognitive tasks. Neuropsychologia, 32, 1443-1452.

Kelley, R. E., Chang, J. Y., Scheinman, N. J., Levin, B. E., Duncan, R. C., \& LEE, S.-C. (1992). Transcranial Doppler assessment of cerebral flow velocity during cognitive tasks. Stroke, 23, 9-14.

Kelley, R. E., Chang, J. Y., Suzuki, S., Levin, B. E., \& ReyesIGLESIAS, Y. (1993). Selective increase in the right hemisphere transcranial Doppler velocity during a spatial task. Cortex, 29, 45-52.

Knecht, S., Henningsen, H., Deppe, M., Huber, T., Ebner, A., \& RINGELSTEIN, E.-B. (1996). Successive activation of both cerebral hemispheres during cued word generation. NeuroReport, 7, 820-824.

MarKus, H. S., \& Boland, M. (1992). "Cognitive activity" monitored by non-invasive measurement of cerebral blood flow velocity and its application to the investigation of cerebral dominance. Cortex, 28, 573-581.

MiLler, N. E. (1995). Clinical-experimental interactions in the development of neuroscience. American Psychologist, 50, 901-911.

NJemanze, P. C. (1991). Cerebral lateralization in linguistic and nonlinguistic perception: Analysis of cognitive styles in the auditory modality. Brain \& Language, 41, 367-380.

Nuemanze, P. C., Gomez, C. R., \& Horenstein, S. (1992). Cerebral lateralization and color perception. Cortex, 28, 69-75.

O'Dell, D. M., RoberTs, A. E., \& MCKINNEY, W. M. (1992a). TCD neurosonology: A window to view thinking. Bulletin of the Psychonomic Society, 30, 237-240.

O'Dell, D. M., RoberTs, A. E., \& McKinney, W. M. (1992b). Transcranial Doppler monitoring of MCA blood flow velocities during three memory tasks. Journal of Neuroimaging, 2, 186-189.

Petersen, S. E., Fox, P. T., Posner, M. I., Mintun M. [A.], \& RAICHLE, M. E. (1988). Positron emission tomographic studies of the cortical anatomy of single-word processing. Nature, 331, 585-589.
Posner, M. I., Petersen, S. E., Fox, P. T., \& Raichle, M. E. (1988). Localization of cognitive operations in the human brain. Science, 240, 1627-1631.

REUTERN, G.-M. voN, \& Büdingen, H. I. von (1993). Ultrasound diagnosis of cerebrovascular disease. New York: Thieme Medical Publishers.

Rihs, F., Gutbrod, K., Gutbrod, B., Steiger, H.-J., Sturzenegger, M., \& MATTLE, H. P. (1995). Determination of cognitive hemispheric dominance by "stereo" transcranial Doppler sonography. Stroke, 26, 70-73.

Schmelzeis, M. C., RoberTs, A. E., \& McKinNeY, W. M. (1994). Cognitive activities modulate cerebral hemovelocities. Psychobiology, 22, 254-258.

Silvestrini, M., Cupini, L. L., Matteis, M., Troisi, E., \& CaltaGIRONE, C. (1994). Bilateral simultaneous assessment of cerebral flow velocity during mental activity. Journal of Cerebral Blood Flow \& Metabolism, 14, 643-648.

Sorteberg, W., Langmoen, I. A., LindegaArd, K. F., \& Nornes, H. (1990). Side-to-side differences and day-to-day variations of transcranial Doppler parameters in normal subjects. Journal of Ultrasound in Medicine, 9, 403-409.

TIPPETT, L. J. (1992). The generation of visual images: A review of neuropsychological theory. Psychological Bulletin, 112, 415-432.

TulviNG, E. (1989). Remembering and knowing the past. American Scientist, 77, 361-369.

VANDENBERG, S. G., \& KUSE, A. R. (1978). Mental rotations, A group test of three-dimensional spatial visualization. Perceptual \& Motor Skills, 47, 599-604.

VARnadore, A. E., Roberts, A. E., \& MCKinNey, W. M. (1997a). Modulations in cerebral hemodynamics under three response requirements while solving language-based problems: A transcranial Doppler study. Neuropsychologia, 35, 1209-1214.

VARNADORE, A. E., RoBerTS, A. E., \& MCKINNEY, W. M. (1997b). The response requirements of nonlanguage problems modulate cerebral hemodynamics: A transcranial Doppler study. In J. Klingelhöfer, E. Bartels, \& E. B. Ringelstein (Eds.), New trends in cerebral hemodynamics and neurosonology (pp. 541-547). New York: Elsevier.

WAINER, H. (1991). Adjusting for differential base rates: Lord's paradox again. Psychological Bulletin, 109, 147-151.

(Manuscript received February 18, 1997; revision accepted for publication February 17, 1998.) 